ITEP-TH-22/08

\title{
Flux-Tube Formation and Holographic Tunneling
}

\author{
A.S. GorSkY ${ }^{1}$ AND V.I. ZaKharov ${ }^{1,2}$ \\ 1- Institute of Theoretical and Experimental Physics \\ B. Cheremushkinskaya ul. 25, 117259 Moscow, Russia \\ 2- INFN - Sezione di Pisa, Largo Pontecorvo, 3, 56127 Pisa, Italy
}

\begin{abstract}
We consider correlator of two concentric Wilson loops, a small and large ones related to the problem of flux-tube formation. There are three mechanisms which can contribute to the connected correlator and yield different dependences on the radius of the small loop. The first one is quite standard and concerns exchange by supergravity modes. We also consider a novel mechanism when the flux-tube formation is described by a barrier transition in the string language, dual to the field-theoretic formulation of Yang-Mills theories. The most interesting possibility within this approach is resonant tunneling which would enhance the correlator of the Wilson loops for particular geometries. The third possibility involves exchange by a dyonic string supplied with the string junction. We introduce also t'Hooft and composite dyonic loops as probes of the flux tube. Implications for lattice measurements are briefly discussed.
\end{abstract}




\section{Introduction}

The interior of the chromomagnetic string connecting external heavy quarks is a traditional subject of theoretical studies devoted to non-perturbative QCD. There are well known phenomena like logarithmic broadening of the string which can be derived from general grounds $[1,2]$. The broadening follows from the account of quantum effects on the string worldsheet which provide the logarithmic dependence of the string width on the length of the string $L, L \gg \Lambda_{Q C D}^{-1}$, with $L$ playing the role of an infrared parameter.

However, as far as dependence on the ultraviolet parameter, say the lattice spacing $a$, is concerned it is assumed, at least tacitly, to be absent. True, the perturbative UV/IR mixing is quite common in the noncommutative gauge theories (for review see [3]) and some features of the noncommutative case could be echoed in the commutative theories as well. However, in the context of the structure of the confining strings such ideas have never been pursued in the literature and the results of the lattice measurements [4] came as a surprise. Namely, according to [4] the width of the flux tube between external quarks depends on the lattice spacing and, if the pattern observed persists at smaller lattice spacings, it vanishes in the continuum limit of $a \rightarrow 0$.

The width of the flux tube is defined in terms of the correlator $\langle s(x), W(T, R)\rangle$ where $s(x)$ is the action density and $W(T, R)$ is the rectangular Wilson loop of space and time extensions $R$ and $T$, respectively. In the lattice language, the point ' $x$ ' is represented by a cube of linear size $a$. In the continuum limit, it is not possible to evaluate the $a$ dependence since the lattice spacing serves as an ultraviolet cut off. Moreover, in the context of the problem considered the lattice spacing $a$ is to be treated rather as an uncertainty in position measurements, or the size of a measuring device [5]. To imitate the problem in the continuum limit we will concentrate, therefore, on the correlator of two concentric round Wilson loops in the adjoint representation,

$$
C\left(R_{2}, R_{1}\right)=\left\langle W_{R_{2}}, W_{R_{1}} ; h=0\right\rangle_{\text {connected }} .
$$

with the radii $R_{2}, R_{1}$ such that $R_{2} \gg R_{1}$, and we fix for simplicity the distance between the circles planes $h=0$. Moreover, since we are aiming at comparison with the lattice data, the space-time is Euclidean.

Once one of the radii, $R_{2}$ is of order $\Lambda_{Q C D}^{-1}$ or larger, the field theoretic formulation is not of much help. Since long [1] one relies instead on a string picture. Thus, we are invited to consider strings in curved space. Note that within this framework the correlator (1) was considered in detail in Ref. [6] (independently of the problem of evaluating the profile of the flux tube). In particular, it was found that a well defined surface with minimal area exists only as far as the ratio $R_{2} / R_{1}$ is not too large. This is an example of a "phase transition" first considered in the stringy setup in Ref. [7]. Beyond a critical value of this ratio the correlator (1) cannot be evaluated in terms of the minimal area of surfaces spanned on the both Wilson loops $W_{1}, W_{2}$.

Since we are interested in very large values of the ratio $R_{2} / R_{1}$ we are well above the "phase transition" point of Ref. [6]. To handle with this regime we have to recognize the mechanism which would provide the connected part in the correlator (1). We shall discuss three possibilities. The first one is quite conventional and was discussed in a different context in [8]. Namely, one considers propagation of the lightest supergravity mode in the bulk between two "cups" of the minimal surfaces in the AdS geometry ending on the small and large circles. One expands the small Wilson loop into a set of local operators and evaluates the correlator of the concentric Wilson loops in terms of the local operators, like in $[8,9,10]$ 
The second possibility we shall also discuss is more tricky. There is no classical minimal surface in the Euclidean space-time above the critical ratio $R_{2} / R_{1}$. However, there exists a complexaction solution to the equations of motion which involves evolution both in the Minkowski and Euclidean spaces. Such solutions in the complex time are quite familiar, say, in the cosmology in the context of the no-boundary Hartle-Hawking wave function. In a more closely related setup, complex-time solutions have been considered in the problem of the multiparticle production on the threshold where they serve as a generating function for the corresponding production amplitudes [11]. What is important is that such complex solutions are intimately related to existence of extended objects and in this sense we are dealing with a purely stringy phenomena. The evolution of such complex solution involves several regions. First it goes in the Euclidean space, then at some "time" it jumps into the Minkowski space-time, undergoes a nontrivial evolution there and then goes back to the Euclidean space. Let us emphasize that the "time", variable here corresponds to the $\mathrm{RG}$ scale.

The most subtle point concerns the Minkowski part of the evolution which in the case considered in [11] is interpreted in terms of the semiclassical bubbles of spherical worldsheets. To ensure stability of such objects the Bohr-Sommerfiedl quantization condition was imposed on the Minkowski bubbles and it turns out that account of such semiclassically quantized bubbles drastically modifies the probability when the boundary conditions in the Euclidean region correspond to the resonance condition fixed by the Bohr-Sommerfield quantization in the Minkowski region. Actually this is an example of the so-called resonant tunneling phenomenon, well known in solid-state physics (see also recent discussion in [12]). Its essence is very simple: if there exist two barriers with a classically allowed region between them the tunneling through the barriers becomes unsuppressed if the energy of the incoming particle coincides with the energy of a metastable state between two barriers. We shall discuss possible resonant tunneling phenomena in our case and derive the resonance condition.

Another possible mechanism concerns magnetic or dyonic strings and their junctions. One can ask if there exists a minimal surface in the Euclidean space-time providing connectness of the Wilson loops which involves the string junctions. We argue that there is such a solution involving propagator of the dyonic string in the bulk. The dyonic string is attached to the small and large Wilson loops by junctions. We will briefly explore more general correlators of the Wilson and t'Hooft loops as well as of the composite loops made of arcs of Wilson and t'Hooft lines.

The organization of the paper is as follows. In Section 2 we shall briefly review phenomenology of the flux tube. In Section 3 we consider the possible minimal surfaces in the AdS geometry and discuss exchange by the supergravity modes. In Section 4 we consider the complex-time solutions and discuss the possibility of resonant tunneling. Section 5 is devoted to the consideration of the mechanism of connectness via dyonic string exchange. In Section 6 we discuss more general configurations involving Wilson, t'Hooft and composite loops.

\section{Overview of the standard flux-tube phenomenology}

For the purpose of orientation, let us make first a few simple phenomenological remarks on the flux tube. On the lattice the intensity of non-Abelian fields inside the flux tube is defined as follows:

$$
\Delta s(x ; R, a) \equiv\langle s\rangle_{0}-\langle s\rangle_{W} \equiv
$$




$$
\langle s\rangle_{0}-\frac{\langle s(x), W(T, R)\rangle}{\langle W(T, R)\rangle},
$$

where $W(T, R)$ is the Wilson line of the (Euclidean) time- and space- extensions $T$ and $R$, respectively, $s$ is the action density in lattice units,

$$
s=a^{4}\left(G_{\mu \nu}^{a}\right)^{2}
$$

$\langle s\rangle_{0}$ is the vacuum expectation value of the action, $\langle($ plaquette $)\rangle \equiv 1-\langle s\rangle_{0}$.

Note that for small lattice spacings the vacuum expectation value of the gluon condensate is divergent,

$$
\left\langle G^{2}\right\rangle_{0} \sim a^{-4}
$$

as a manifestation of the uncertainty principle. On the other hand, the common expectation for the confining fields inside inside the tube is that they are soft,

$$
\left\langle G^{2}\right\rangle_{\text {soft }} \sim \Lambda_{Q C D}^{4}
$$

In other words, the difference of the plaquette values (2) is expected to be vanishing in the limit $a \rightarrow 0$ :

$$
a^{4}\left\langle G^{2}\right\rangle_{0}-a^{4}\left\langle G^{2}\right\rangle_{W} \sim\left(a \cdot \Lambda_{Q C D}\right)^{4}
$$

Note that $\Delta s(R, a)$ is positive, as guaranteed by the exact sum rules for the string tension [13]. Moreover, the difference (2) is usually fitted to a Gaussian form:

$$
\Delta s(x ; R, a) \sim \exp \left\{-h^{2}(x) / \delta^{2}(R, a)\right\}
$$

where $h(x)$ is the distance from the point $x$ to the plane determined by the Wilson loop, and $\delta$ is the width of the flux tube. It goes without saying that the standard picture assumes that the width is independent on the lattice spacing, $\delta \sim \Lambda_{Q C D}^{-1}$, and one usually concentrates on the infrared, or $R$-dependence of the width $[1,2]$.

On the other hand, recent lattice measurements [4] found out that in fact the width (4) depends drastically on the lattice spacing which corresponds to the dependence on $a$ different from (3). This observation is in contradiction with the standard picture and our aim here is to investigate whether string-related phenomenology could accommodate the observation [4].

\section{Correlator of concentric Wilson lines. Generalities.}

It is well known nowadays that the dual description of gauge theories is provided by strings in curved space. In particular, in the conformal case the metric is:

$$
d s^{2}=R_{A d S}^{2} \frac{1}{z^{2}}\left(d x_{i}^{2}+d z^{2}\right)
$$

The expectation value of the Wilson loop is controlled by the minimal value of the area of surface spanned on the loop [14]. One can then readily see that the effective action, or area is given by

$$
S=2 \pi R_{A d S}^{2}\left(\int d z \frac{r}{z^{2}} \sqrt{1+\left(r^{\prime}\right)^{2}}\right)
$$


where the limits of integration over $z$ are set by boundary conditions, or the shape of the Wilson loops. The equation of motion, corresponding to the action (6) reads as:

$$
\frac{d}{d z} \frac{r r^{\prime}}{z^{2} \sqrt{1+\left(r^{\prime}\right)^{2}}}=\frac{\sqrt{1+\left(r^{\prime}\right)^{2}}}{z^{2}} .
$$

The solution for the minimal area spanned on a circular Wilson loop, relevant to our case, is well known. To derive the solution, it is convenient to introduce $(\rho, \tau)$ coordinates instead of $(z, r)$, where $r$ is the radial coordinate on the boundary:

$$
z=e^{\tau} \cosh ^{-1} \rho \quad r=e^{\tau} \tanh \rho
$$

This substitution makes the conformal symmetry manifest, and one can readily find an integral of motion which exists as a reflection of this symmetry. Denoting the value of this integral of motion as $c$, one can derive an equation for the minimal surface in terms of this constant $c$ :

$$
\int d \tau= \pm c \int \frac{d \rho}{\cosh \rho \sqrt{\sinh ^{2} \rho \cosh ^{2} \rho-c^{2}}}
$$

where the limits of integration are set by the boundary conditions.

For a single circular Wilson loop the minimal surface is given by the solution $\tau=$ const so that the left-hand side of Eq. (9) vanishes and, therefore, $c=0$. The corresponding action, or area is given by

$$
S_{R}=R_{A d S}^{2}\left[\frac{R}{\epsilon}-1\right]
$$

where $\epsilon$ is an ultraviolet cut off needed to regularize divergence in action (6) at $z \rightarrow 0$. The divergent term is proportional to the perimeter of the Wilson line and corresponds to the self energy of the external W-boson.

The case of two concentric Wilson loops was studied first in Ref. [6], see also [15]. For us the central point is that minimal surface can be determined only if the ratio $R_{2} / R_{1}$ is not too large. Knowing the condition (9) allows to readily derive this result. Indeed, we need now $z \rightarrow 0$ both for $r=R_{1}$ and $r=R_{2}$. Moreover, $z \rightarrow 0$ implies $\tanh \rho=1$. Then the left hand side of Eq. (9) is equal to $\ln \left(R_{2} / R_{1}\right)$. On the other hand the right-hand side of Eq. (9) is limited by a quantity of order unit, for any $c$. Thus, large $\ln \left(R_{2} / R_{1}\right)$ is not allowed. More details can be found in $[6,15]$.

Let us first consider the mechanism of connectness above the critical point via exchange by supergravity modes. That is we shall discuss the configuration of two cups corresponding to small and large Wilson loops. They are connected through the exchange by supergravity modes described by bulk-bulk propagators. In our case, the calculation can be simplified since one of the Wilson loops is very small. That is, it is natural to replace it by a local operator and therefore the bulk-bulk propagator can be replaced by a bulk-boundary one. Hence we arrive at the problem of calculation of the correlator of local operator with the large circular Wilson loop. Such a problem has been discussed before in $[8,9]$.

We substitute the small Wilson loop by a sum over local operators $O_{N}$ with canonical dimensions $\Delta_{N}$ :

$$
W\left(R_{1}\right)=<W\left(R_{1}\right)>\sum_{N} c_{N}\left(R_{1}\right) O_{N}, \quad c_{N} \propto R_{1}^{\Delta_{N}}
$$


and select the operator with the minimal dimension $O=\operatorname{Tr} G^{2}$ which couples to the dilaton mode in the bulk. The mass of the bulk mode coupled to the operator with dimension $\Delta$ in $A d S_{5}$ reads as

$$
m^{2}=\Delta(\Delta-4)
$$

that is the dilaton is a massless mode in the AdS bulk. The correlator reduces to the product of the bulk-boundary propagator of the dilaton and of the vacuum expectation value of the dilaton vertex operator integrated over the string worldsheet with the fixed boundary condition on the large circle of radius $R_{2}$. Since we are interested in the $R_{1}$ dependence of the correlator this one-point function on the worldsheet provides the $R_{1}$ independent multiplier which could be calculated similar to $[8,9]$. The bulk-boundary propagator evidently does not depend on $R_{1}$ as well hence we arrive at the following form of the correlator

$$
<W\left(R_{1}\right), W\left(R_{2}\right)>\propto \frac{R_{1}^{4}}{R_{2}^{4}} F\left(R_{2}\right)
$$

with a calculable function $F\left(R_{2}\right)$.

So far we discussed the conformal case which admits for exact solutions. Generalizations to the realistic QCD case require for extra assumptions. For the sake of estimates, we can use a simple model with a phenomenologically motivated modification of the metric (5) which explicitly introduces the $\Lambda_{Q C D}$ scale.

In particular to get qualitative estimates, one may have in mind, for example, the following modification of the metric (5):

$$
\frac{1}{z^{2}} \rightarrow \frac{1}{z^{2}} \exp \left(\text { const } \cdot z^{2}\right)
$$

where const $\sim \Lambda_{Q C D}^{2}$, see, e.g. [16] and references therein.

The crucial point is that at short distances the metric (14) is still conformal nd one can still rely on the derivations valid in the conformal space. On the other hand, the modification (14) introduces an infrared cut off at $R_{2} \sim \Lambda_{Q C D}^{-1}$. Thus, for the sake of estimates in the realistic case one can rely on the conformal-case relations provided the following substitution is made:

$$
R_{1} \rightarrow a, \quad R_{2} \rightarrow \Lambda_{Q C D}^{-1} \quad, \quad R_{2} / R_{1} \rightarrow\left(\Lambda_{Q C D} \cdot a\right)^{-1}
$$

With this rule in hand, one readily derives predictions for the realistic case.

\section{AdS geometry, complex time solutions and resonant tunneling}

In the absence of the minimal surface with our boundary conditions in the Euclidean space we could ask if there is a solution to the equation of motion in the complex "time". Remind that the "time" variable in the problem is in fact the radial Liouville coordinate in a AdS-type geometry. Hence the complex "time" corresponds to the complexified radial coordinate. We shall look for a complex-time solution which involves two Euclidean regions and one Minkowski

region between them. From quantum mechanics we know that such a solution corresponds to tunneling through two barriers with the classically allowed region between them. Indeed, the 
under-barrier region naturally corresponds to the Euclidean region while the interval between the barriers corresponds to the Minkowski space. We shall use this viewpoint below. Note that in the stringy Schwinger production the breakdown of the minimal surface precisely corresponds to emergence of a classically allowed production in the Minkowski space [18], supporting this general picture.

Let us start with a simplified case and tend, for a moment, $R_{1} \rightarrow 0$ and $R_{2} \rightarrow \infty$. Then there is no scale left in the problem and one is invited to look for a solution

$$
r=C \cdot z
$$

where $C$ is a constant. Substituting ansatz (16) into the equation of motion (7) one finds:

$$
C^{2}=-1 / 2
$$

and the respective action (6) is pure imaginary:

$$
S= \pm \sqrt{-1} \pi R_{A d S}^{2}\left(\ln R_{\max } / R_{\min }\right)
$$

where, to regularize the logarithmic divergence of the action, we introduced infrared and ultraviolet cuts off. Obviously, $R_{\max } \approx R_{2}, R_{\min } \approx R_{1}$.

It is worth emphasizing that the solution (16) was recently found and discussed in Ref. [17], in another context. In that case one starts with a problem in Minkowski space and finds that the extremal trajectory corresponds to an imaginary action, or Euclidean signature. We start with a problem in Euclidean space and find that the extremal trajectory corresponds to an imaginary action, or Minkowski signature.

The actual extremal trajectory is to be more complicated than (16). Indeed, at very small $z$ there is an ultraviolet divergence in the action, due to the self-energy, see (10). It goes without saying that we should consider $\epsilon \ll R_{1}$. Thus, the extremal trajectory consists of three pieces, all of which are described in terms of real radial coordinate $r$ and either real or imaginary coordinate $z$ :

$$
\begin{array}{r}
\epsilon<r<R_{\min } \sim R_{1}: z \approx \sqrt{R_{1}^{2}-r^{2}} \\
R_{\min }<r<R_{\max } \sim R_{2}: z \approx \sqrt{-2} r \\
R_{\max }<r<R_{2}: z \approx \sqrt{R_{2}^{2}-r^{2}}
\end{array}
$$

Moreover generically one could discuss the dependence of the radius on two coordinates similar to $[6]$.

That is the total action on the trajectory has the following structure

$$
S_{t o t}=S_{e v k l, 1}\left(R_{2}\right)+S_{e v k l, 2}\left(R_{1}\right)+i S_{\text {mink }}\left(R_{1}, R_{2}\right)
$$

where $S_{e v k l, R_{1}}, S_{e v k l, 2}$ correspond to the actions on the cups and the imaginary contribution corresponds to the Minkowski region. As we have seen the imaginary contribution involves the logarithmic dependencies on the radii. Naively, the imaginary contribution does not influence the exponential factor which depends on $R_{2}$ only. Indeed in the non-conformal case we have area law hence the area of the large loop dominates in the action and there is no $R_{1}$ dependence at all. However from the textbooks on quantum mechanics we know about the phenomena of the 


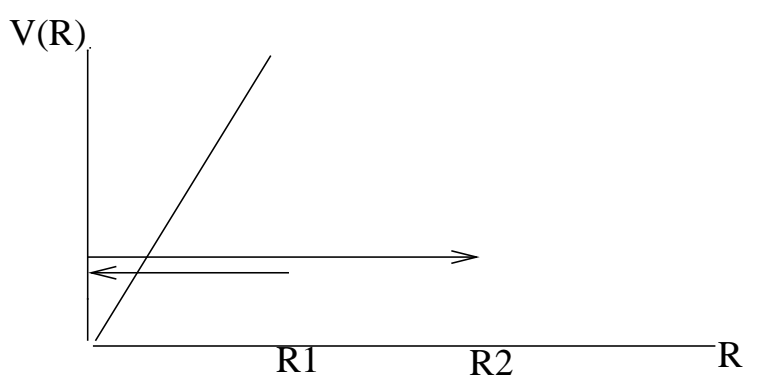

Figure 1. Semiclassical trajectory corresponding to the resonant tunneling

resonant tunneling through two barriers. It happens when the energy of the incoming particle coincides with some level from the WKB spectrum of the metastable states between the barriers.

Let us remind standard facts about the resonant tunneling. Denote the tunneling factors associated with the two barriers as $Z_{1}(E)=\exp \left(-S_{1}\right)$ and $Z_{2}(E)=\exp \left(-S_{2}\right)$. In the generic situation the full tunneling probability is determined by the product $Z_{1} \cdot Z_{2}$. However under the resonance condition the situation changes, the tunneling probability reads as

$$
T=\frac{4}{\left(Z_{1} / Z_{2}+Z_{2} / Z_{1}\right)^{2}}
$$

and in case of symmetric barriers tends to unit. In case of the asymmetric barriers the total probability depends on the ratio of two Euclidean actions. Let us emphasize that the resonant tunneling emerges upon taking into account an infinite number of the oscillations in the Minkowski region.

Actually the problem we consider has a lot in common with the one discussed in the context of multipartucle production [11]. Let us remind that problem and map it to our complex time solution. In that case one considers decay of a highly virtual particle into the many-body state of particles almost at the threshold. It was argued that the problem can be reduced to the resonant tunneling. Namely one has to look for the solution to the classical equations of motion with the Nambu-Goto type action with peculiar boundary conditions. Such a solution has been found in [11] and can be described in 3D case as follows. The initial particle with energy $E$ gets transformed into an expanding closed string in the Minkowski space. Then, at radius $r_{0}$, whose value depends on the energy of the incoming particle this string enters the Euclidean space- time region as the solution with the same energy. The effective potential for this problem behaves as

$$
V(R)=\sigma R
$$

where $\sigma$ is the tension of the string and $\mathrm{R}$ is the radius of the circle.

The key point is that this potential enjoys semiclassical levels which can be easily calculated in the WKB approximation

$$
E_{N} \propto \sqrt{\sigma} N^{2 / 3}
$$

and resonant tunneling happens if $E=E_{N}$, see Figure 1 .

Let us argue that we have quite a similar situation in our problem. Once again in the simplest case the potential for the only dynamical variable $r(z)$ is linear and we may use it to discuss the problem qualitatively. With our boundary conditions we look for the trajectory which starts at the small-loop radius and upon a fixed "time" gets onto the large-loop radius. The breakdown of 
the minimal surface tells us that such boundary conditions can not be fulfilled in the Euclidean region. However they can be satisfied if the evolution partly happens in the Minkowski spacetime similar to the example discussed above. Generically the Minkowskian contribution to the action is imaginary but if the resonance condition is imposed it influences the real part as well. In our case the resonance condition is given by the equation:

$$
S_{\text {mink }}=2 \pi N \propto \sqrt{\lambda} \log \frac{R_{2}}{R_{1}}
$$

where $\mathrm{N}$ is an arbitrary integer number and $\lambda$ is the t'Hooft coupling. The action calculated on the solution is proportional to the tension of the string which in the conformal case is proportional to the Yang-Mills coupling constant $g_{Y M}$. Hence we could rewrite the resonance condition in the form

$$
R_{I R} / R_{U V}=\exp \left(c / g_{Y M}\right)
$$

which resembles the dimensional transmutation formula relating the IR and UV scales in the theory with asymptotic freedom. The coupling dependence is however different which could be interpreted as an indication that a nontrivial function of the coupling constant is involved in nonconformal case which behaves as $1 / g_{Y M}^{2}$ at weak coupling and $1 / g_{Y M}$ at strong coupling. In nonconformal case we can safely identify $R_{I R}$ with $\Lambda^{-1}$.

Provided that this condition (23) is fulfilled, one can expect that there is a metastable level and the probability of the tunneling is not actually suppressed, see (21). This means in turn that there is strong dependence of the correlator (2) on the size of the small Wilson loop, or lattice spacing $a$ in case of lattice measurements.

There are reservations to be made concerning the complex-time solution. In particular, it is unclear if the solution we consider is stable or not, that is if it has a negative mode in the spectrum of fluctuations. It seems probable that it is unstable and therefore describes the "time" decay of the state. Since "time" is our case is identified with the radial RG coordinate then the "decay" would mean the RG flow from UV to IR.

\section{$5 \quad$ Minimal surfaces with the string junctions}

Generically, one can consider t'Hooft loops along with the Wilson loops. The t'Hooft loops are obtained via the D1 string worldsheet in the bulk supported by a contour on the boundary. Let us discuss possible role of the D1 and (p,q) dyonic strings in our problem. The configuration we shall look for involves the tube of the dyonic string connected with the F1 worldsheets via junctions, see Figure 2. That is there are two discs of the magnetic string worldsheets on two cups. To discuss the classical configuration we have to recognize the mechanism of the stabilization of the radii of the discs.

The problem strongly depends on the ratio of the tensions of the F1 and D1 strings. At weak coupling F1 strings certainly are lighter than D1 strings. However at the strong coupling is its not necessarily true and the opposite limit is more reliable. Hence at weak coupling the radius stabilization is impossible for the dyonic tube connecting two cups, as can be seen from the simple energy considerations.Indeed, consider the change of the total action of the solution due to the long tube. There are positive contributions due to the area of the cylinder and the area of the magnetic string discs since it is heavier than the F1 string. That is there is no reason 


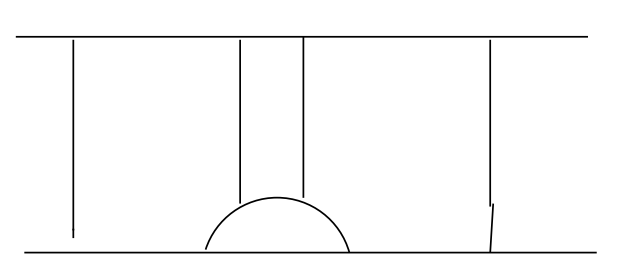

horizon

Figure 2. Geometry of the configuration corresponding to the magnetic string exchange

for the stabilization of the radius of the $(1,1)$ cylinder and it shrinks to zero. Therefore there is no classical solution of such type at the weak coupling.

However, a classical solution seems to exist at the strong coupling limit when the F1 string is heavier then the magnetic string. To identify the mechanism of the stabilization let us first consider the simplest flat case when the competition of two terms in the effective action for the cylinder radius happens:

$$
S_{R}=2 T_{1,1} \pi r H-\left(T_{(1,0)}-T_{(0,1)}\right) \pi r^{2}
$$

where $H$ is the length of the cylinder. That is the stabilization of the radius of the cylinder and, therefore, the solution to the classical equation of motion turn possible. However, there is a restriction on the radius of the circle since it can not exceed the radius of the small cup $a$. Extremizing the effective action yields:

$$
r_{c r}=\frac{H T_{1,1}}{\delta T}
$$

that is we have the condition

$$
\frac{H T_{0,1}}{\delta T} \leq a
$$

At the strong coupling, the length $\mathrm{H}$ in the nonconformal case can be approximated by $\Lambda_{Q C D}^{-1}$ while the difference of the tensions of the strings depends on details of the background. Therefore we see that the mechanism of the connectness of the Wilson loops correlator due to the exchange by the dyonic string tube can work only in some range of fine tuned parameters.

Let us turn to a more interesting case of the AdS-type geometry. We are looking for a solution to the equation of motion with the real action for the dyonic string. We expect that the dyonic string worldsheet is glued with the cups at fixed radius and radial coordinate. That is consider the following anzatz for the solution

$$
\left(r-r_{0}\right)=C\left(z-z_{0}\right)
$$

which slightly generalizes the solution discussed above. The crucial point is that this modification makes the action real, in contradistinction from the imaginary action discussed above. Indeed substituting this anzatz into the equation of motion one gets

$$
C=-\frac{z_{0}}{2 r_{0}}
$$

and the corresponding action reads as

$$
S_{a d s}=C \sqrt{1+C^{2}} \log \frac{R_{\text {Min }}}{R_{\max }}+\ldots
$$


Hence the solution amounts to a power-like dependence on the radius of the small circle. To fix the radii one has to minimize the effective action with respect to $r_{0}, z_{0}$, similar to the consideration in [18]. The action depends on details of the background however the very fact of power-like dependence of the correlator of the Wilson loops on the radius of the small circle is generic, within this mechanism of the connectness.

Let us comment on the impact of the horizon inherent to confining backgrounds. The fundamental string tension at the horizon is of order $\Lambda_{Q C D}^{2}$ while the tension of the magnetic string effectively vanishes. The most simple argument for this tensionless magnetic string goes as follows [19]. Let us assume the approach based on the wrapped D4 branes background [21] and represent the magnetic string by a D2 brane wrapped around the angular circle of the cigar. Below the critical temperature such wrapping is topologically unstable that is D2 brane tends to the tip of the cigar where is tension clearly vanishes. This phenomena is responsible for the perimeter law of the t'Hooft loop in the confining phase. Above the critical temperature the wrapping becomes stable and the magnetic string becomes tensionful. That is below the critical temperature the magnetic string involved into the dyonic mechanism tends to expand at the horizon region supporting the relevance of the solution discussed above.

Another point to be mentioned is that the logarithmic dependence on the small radius follows also from the deformation of the small cup by the dyonic string tension, similar to the case discussed in [18]. The reason for this additional logarithmic terms is simple: they just reflect the behavior of the solution to the Laplace equations with a source in two dimensions.

\section{Correlators involving t'Hooft loops}

Let us consider now the probe of the interior of the large Wilson loop by a t'Hooft or dyonic loop. That is we consider the connected correlator

$$
\frac{<W\left(R_{2}\right) W_{H}\left(R_{1}\right)>}{<W\left(R_{2}\right)><W_{H}\left(R_{1}\right)>}
$$

and discuss the mechanism of connectness via the dyonic string tube. Once again to discuss the classical configuration we have to get the stabilization of the radii of the tube. That is the tensions of the strings with the disc topology has to be smaller than tensions outside the tube.

Consider the weak coupling regime when the F1 string is light and exchange is by the dyonic string. That is the disc at the large cup corresponds to the magnetic string worldsheet and therefore tends to expand and its stabilization by the tension is possible. The disc on the small cup can be stabilized as well since it is made from the F1 string which is lighter than the magnetic string. Hence at least at weak coupling the "dyonic mechanism" is possible and it deserves detailed investigation.

The next example concerns the correlator of the large Wilson loop and a small dyonic Wilsont'Hooft loop

$$
\frac{<W\left(R_{2}\right) W_{D}\left(R_{1}\right)>}{<W\left(R_{2}\right)><W_{D}\left(R_{1}\right)>}
$$

It is easy to see that for the $(1,1)$ loop the mechanism does not work. To get the stabilization of the cylinder we have to find the configuration when two discs at small and large cups are the worldsheets of magnetic strings. Taking into account the charge conservation at the string junctions we find, somewhat surprisingly, that the stabilization can happen only if we consider 


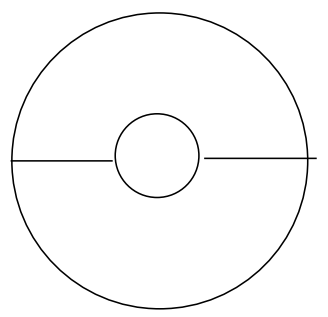

Figure 3. The boundary slice of the

configuration corresponding to the composite loops

the correlator of the small Wilson loop of the $(1,2)$ type with at least magnetic charge two. Then the tube is the worldsheet of dyonic $(1,1)$ string and the two circles are worldsheets of D1 strings.

One has the restriction that the small radius at equilibrium is to be smaller than the radius of the small loop $a$ which yields the restriction on the possible set of parameters. Of course the consideration of this section is at a qualitative level and a more detailed analysis is required to confirm the possibility of this mechanism.

Finally let us consider more exotic composite Wilson-t'Hooft loops made from two segments, one "Wilson segment" and one "t'Hooft segment". Since the tensions of F1 and D1 strings and their charges are different the junction of two segments is nontrivial and has to be accomplished by an additional $(1,1)$ dyonic string.

For two parallel Wilson and t'Hooft lines which measure the quark-monopole interaction such worldsheet involving string junction has been considered in [20] where the corresponding minimal surface consideration has been found. Here we consider the generalization of the similar picture for the case of two concentric composite loops.

Due to the local equilibrium condition at the junction line and the charge conservation one has to consider the geometry involving two "composite cups" and the worldsheet of the $(1,1)$ dyonic string between them. The qualitative picture of the string geometry at the boundary is presented at Fig.3. The action for the configuration reads as follows

$$
S=A_{\text {big }}+A_{\text {dyonic }}+A_{\text {small }}
$$

where three terms correspond to the area of the small, big cups and the area of the dyonic worldsheet between two cups. In the nonconformal case one could expect that the first term yields the "electric" area term while the second yields "magnetic" perimeter term. Let us note that dyonic worldsheet can be oriented differently in the Euclidean space-time. If there is negative mode on this composite minimal surface then these orientations yield the different final states upon the decay process. If there are no negative mode it is natural to integrate over the orientation of the dyonic string worldsheet which yields only inessential numerical factor.

\section{Conclusions}

In the paper we have discussed the qualitative picture behind the connected correlator of two concentric Wilson loops in the adjoint representation with the very different radii. We have mainly considered three mechanisms possibly determining intensity of non-Abelian fields inside the flux tube. The first one is exchange of the dilaton in bulk. It results in the prediction

$$
\langle s\rangle_{0}-\langle s\rangle_{W} \sim\left(\Lambda_{Q C D} \cdot a\right)^{4},
$$


where $a$ is the size of the probe, or lattice spacing and the power of the factor $\left(\Lambda_{Q C D} \cdot a\right)$ is fixed by the properties of the dilaton mode in the conformal limit. Thus, the dilaton exchange reproduces, in the dual language, the "common-sense" expectation (3) that the fields inside the flux tubes are soft. This prediction (34) does not appear self-contradictory by itself. However, it cannot accommodate recent lattice observation [4] that the distribution of the fields inside the tube depends crucially on the size of the probe.

There is another possible mechanism determining the correlator of the two Wilson loops which is tunneling in terms of the dual description. The extremal trajectory involves evolution both in Euclidean and Minkowski spaces and corresponds to complex-time solutions in quantum mechanics. From quantum-mechanical examples it is known, furthermore, that such solutions can produce resonant-type dependence for the correlator on the radii of the circles. We have a kind of parametric resonance, typical for nonlinear systems.

Invoking this mechanism would allow to accommodate strong dependence on the size of the small Wilson loop discovered in the lattice measurements [4]. However, at this moment it would be premature to make a strong claim of 'explaining' the lattice data or performing detailed fits of the data. Instead, we confine ourselves to a remark that further checks are possible on the lattice. In particular, a prediction is that dependence on the lattice spacing is of resonance-type.

We have also qualitatively discussed the possible role of the configurations involving the string junctions. It was argued that such classical configuration yields the power dependence on the radius of the small circle. The corresponding power depends on the details of the nonconformal background. We have also introduced the interesting objects - composite Wilson-t'Hooft loops which can not exist separately but there is natural "bound state" of two such composite loops which certainly deserves for the further investigation.

Let us mention a few questions which could be also analyzed within our approach. The first point concerns the temperature dependence which strongly influences the dyonic mechanism since the magnetic string becomes tensionful above the phase transition point. Another generalization concerns the Wilson and t'Hooft loops in the different representations. The case of fundamental representation could be of the special interest since it could be responsible for the bound states of the different mesons. The example of such bound state via the bulk dilaton exchange has been found recently in [22]. The Wilson loops in the symmetric and antisymmetric representations are treated in terms of dielectric five-branes and deserves for the separate analysis.

A.G. thanks INT at University of Washington where the part of the work has been done during the Workshop "From Strings to Things" for the hospitality and support. The work of A.G. was supported in part by grants RFBR-06-02-17382, INTAS-1000008-7865, PICS- 07-0292165.

\section{References}

[1] M. Luscher, G. Munster, P. Weisz, 'How Thick Are Chromoelectric Flux Tubes?", Nucl. Phys. B180 (1981) 1.

[2] J. Greensite, P. Olesen, "Broadening of the QCD(3) flux tube from the AdS/CFT correspondence", JHEP 0011 (2000) 030, [arXiv:hep-th/0008080];

A. Loewy, J. Sonnenschein, "On the holographic duals of $N=1$ gauge dynamics", JHEP 0108 (2001) 007, [arXiv:hep-th/0103163]. 
[3] M.R. Doiuglas, N.A. Nekrasov, "Noncommutative field theory", Rev. Mod. Phys., 73, (2001) 977, [arXiv:hep-th/0106048].

[4] P.Yu. Boyko, F.V. Gubarev, S.M. Morozov, "On the fine structure of QCD confining string", PoS LAT2007 307,(2007) 307, e-Print: arXiv:0712.0656 [hep-lat].

[5] V.I. Zakharov, "Matter of resolution: From quasiclassics to fine tuning", ArXiv:hepph/0602141; "Nonperturbative physics at short distances", arXiv:0705.0743 [hep-ph].

[6] P. Olesen , K. Zarembo, "Phase transition in Wilson loop correlator from AdS / CFT correspondence", [arXiv:hep-th/0009210].

[7] D. J. Gross and H. Ooguri, "Aspects of large N gauge theory dynamics as seen by string theory," Phys. Rev. D 58, 106002 (1998) [arXiv:hep-th/9805129].

[8] D. Berenstein, R. Corrado, W. Fischler, J.M. Maldacena, " The Operator product expansion for Wilson loops and surfaces in the large N limit", Phys. Rev. D59 (1999) 105023, [arXiv:hep-th/9809188];

N. Drukker , D.J. Gross , H. Ooguri, "Wilson loops and minimal surfaces", Phys. Rev. D60 (1999) 125006, arXiv:hep-th/9904191.

[9] K. Zarembo, "Open string fluctuations in $A d S(5) \times S(5)$ and operators with large R charge", Phys. Rev., D66 (2002) 3843 .

[10] I. R. Klebanov, J. M. Maldacena and C. B. . Thorn, "Dynamics of flux tubes in large N gauge theories," JHEP 0604, 024 (2006) [arXiv:hep-th/0602255].

[11] A. S. Gorsky, M. B. Voloshin, "Nonperturbative production of multiboson states and quantum bubbles", Phys. Rev. D48, (1993) 3843, [arXiv:hep-ph/9305219].

[12] P. M. Saffin, A. Padilla and E. J. Copeland, "Decay of an inhomogeneous state via resonant tunnelling," arXiv:0804.3801 [hep-th].

[13] C. Michael, "Lattice Action Sum Rules", Nucl. Phys. B280 (1987) 13;

H.J. Rothe, "Lattice energy sum rule and the trace anomaly", Phys. Lett. B364 (1995) 227, arXiv:hep-lat/9508005.

[14] J. M. Maldacena, "Wilson loops in large N field theories," Phys. Rev. Lett. 80, 4859 (1998) [arXiv:hep-th/9803002].

S. J. Rey and J. T. Yee, "Macroscopic strings as heavy quarks in large N gauge theory and anti-de Sitter supergravity," Eur. Phys. J. C 22, 379 (2001) [arXiv:hep-th/9803001].

[15] T. Hirata, T. Takayanagi, "AdS/CFT and strong subadditivity of entanglement entropy", JHEP 0702 (2007) 042, arXiv:hep-th/0608213.

[16] A. Karch, E. Katz, D. T. Son and M. A. Stephanov, "Linear confinement and AdS/QCD," Phys. Rev. D 74, 015005 (2006) [arXiv:hep-ph/0602229].

O. Andreev , V.I. Zakharov, "Heavy-quark potentials and AdS/QCD”, Phys. Rev. D74 (2006) 025023, [arXiv:hep-ph/0604204]. 
[17] L.F. Alday, J. Maldacena, "Gluon scattering amplitudes at strong coupling",JHEP 0706 (2007) 064, arXiv:0705.0303 [hep-th].

[18] A.S. Gorsky, K.A. Saraikin, K.G. Selivanov, Schwinger type processes via branes and their gravity duals", Nucl. Phys. B628 (2002) 270, arXiv:hep-th/0110178.

[19] A. Gorsky and V. Zakharov, "Magnetic strings in Lattice QCD as Nonabelian Vortices," Phys. Rev. D 77, 045017 (2008) [arXiv:0707.1284 [hep-th]].

[20] J. A. Minahan, "Quark-monopole potentials in large N super Yang-Mills," Adv. Theor. Math. Phys. 2, 559 (1998) [arXiv:hep-th/9803111].

[21] E. Witten, "Anti-de Sitter space, thermal phase transition, and confinement in gauge theories," Adv. Theor. Math. Phys. 2, 505 (1998) [arXiv:hep-th/9803131].

[22] S. Dubynskiy, A. Gorsky and M. B. Voloshin, "Holographic Hadro-Quarkonium," arXiv:0804.2244 [hep-th]. 